\title{
RELATIVIST IC FREE-FREE GAUNT FACTOR OF THE DENSE HIGH-TEMPERATURE STELLAR PLASMA
}

Naoki Itoh, Masayuki Nakagawa, and Yasuharu Kohyama

Department of Physics, Sophia University, Tokyo, Japan

ABSTRACT The free-free Gaunt factor of the dense hightemperature stellar plasma is calculated by using the accurate relativistic cross section, and is compared with the Gaunt factor derived by using Sommerfeld's exact nonrelativistic cross section. A wide range of electron degeneracy is accurately taken into account. Significant deviations from the nonrelativistic relsults are found for high-temperature cases.

\section{I NTRODUCT I ON}

The present authors (Itoh, Nakagawa, and Kohyama 1985) have investigated the relativistic free-free Gaunt factor for a high-temperature stellar plasma. They have used the relativistic Bethe-Heitler cross section (Bethe and Heitler 1934) corrected by the Elwert factor (Elwert 1939). I toh, Nakagawa, and Kohyama ( 1985 ) have found that the relativistic Gaunt factor deviates significantly from the nonrelativistic one at high temperatures.

The exact nonrelativistic bremsstrahlung cross section for the pure Coulomb field is given by Sommerfeld's formula (Sommerfeld 1953 ). For practical calculations we use the series expantion method of Karzas and Latter (1961) by taking into account the electron degeneracy accurately. Green ( 1960 ) has also given numerical tables of the exact nonrelativistic free-free Gaunt factor taking into account the electron degeneracy accurately. Berger (1956, 1957) has examined the accuracy of the Elwert approximation. However, his calculation is limited to the case of nonrelativistic nondegenerate electrons. Elwert and Haug (1969) and Pratt and Tseng( 1975) has confirmed that the Bethe-Heitler cross section corrected by the Elwert factor gives excellent results for ions with small atomic number.

\section{FORMULAT ION}

The detailed derivation of the relativistic free-free Gaunt factor with the use of the Elwert approximation has been presented in Iton, Nakagawa, and Kohyama ( 1985 ). 
When the electrons are nonrelativistic, it is customary to express the thermally averaged inverse bremsstrahlung cross section in terms of the thermally averaged Kramers cross section per electron,

$$
\begin{aligned}
\sigma_{K} & =\frac{2}{3}\left(\frac{2 m c^{2}}{3 \pi k T}\right)^{1 / 2} \frac{(2 \pi c)^{3}}{\omega^{3}} \alpha r_{0}^{2} n_{j} z_{j}^{2} \\
& =\frac{16 \sqrt{2}}{3 \sqrt{3}} \pi^{5 / 2} \alpha r_{0}{ }^{2} \frac{m^{1 / 2} h^{3} c^{4}}{(k T)^{7 / 2} u^{3}} n_{j} z_{j}^{2},
\end{aligned}
$$

where $m$ is the electron mass, $\omega$ is the angular frequency of the absorbed photon, $\alpha$ is the fine-structure constant, $r_{0}$ is the classical electorn radious, $n_{j}$ is the number density of ions with charge $Z_{j} e$, and $u=\hbar \omega / k T$. We define the relativistic free-free Gaunt factor by

$$
\left\langle g_{Z_{j}}\right\rangle=\frac{\left.n_{-}\left\langle\sigma^{-}\right\rangle+n_{+}<\sigma^{+}\right\rangle}{n_{j} Z_{j}\left\langle\sigma_{k}>\right.},
$$

where $n_{-}$and $n_{+}$are the number densities of electrons and positrons, and $\left\langle\sigma^{-}\right\rangle$and $\left\langle\sigma^{+}\right\rangle$are the thermally averaged inverse bremsstrahlung cross sections for the electrons and the positrons. The thermal averages of the these cross sections are carried out relativistically.

Green ( 1960 ) calculated the thermally averaged exact nonrelativistic free-free Gaunt factor for the case of degenerate electrons by using the energy-dependent Gaunt factor of Karzas and Latter (1961). In using their data, he had to make interpolations with respect to $u=\hbar \omega / \mathrm{kT}$. Nakagawa, Kohyama, and Itoh ( 1987 ) calculated the exact nonrelativistic energy-dependent free-free Gaunt factor, and then used this for the calculation of the thermally averaged Gaunt factor without making any interpolation.

\section{NUMERICAL RESULTS}

We have carried out the numerical calculations of the thermally averaged nonrelativistic and relativistic freefree Gaunt factors. The temperature parameter

$$
r^{2}=\frac{Z_{j}^{2} R y}{k T}=Z_{j} 2 \frac{1.579 \times 10^{5} \mathrm{~K}}{T}
$$

is adopted following Karzas and Latter ( 1961 ) and Green $(1960)$. For the nonrelativistic case, the thermally averaged free-free Gaunt factor does not depend on $Z_{j}$ and $T$ separately, but on the combination $Z_{j} 2 / T$. Examples of the 
calculations are shown in Figures 1 and 2. Agreement of the results of the calculations with different methods proves the accuracy of the present calculations. The overall accuracy of the present calculations is about $0.2 \%$.

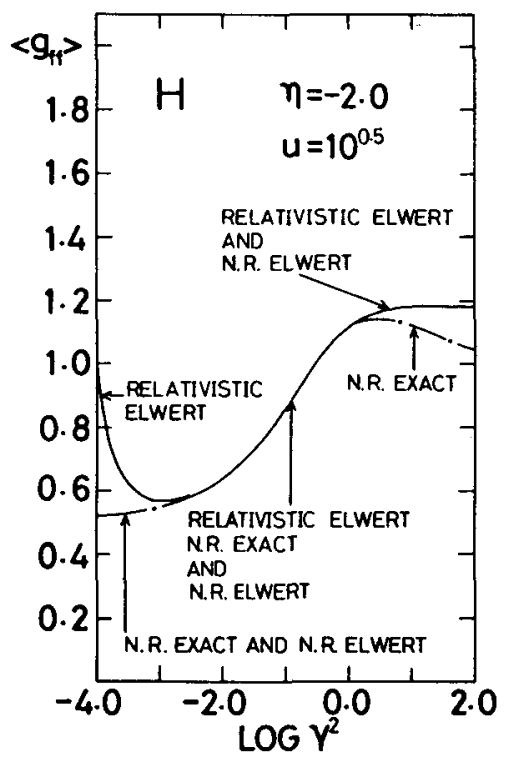

FIG. 1. Comparison of the various thermally averaged freefree Gaunt factors for the case of pure hydrogen plasma with $\eta=-2.0$ and $u=10^{0.5}$.

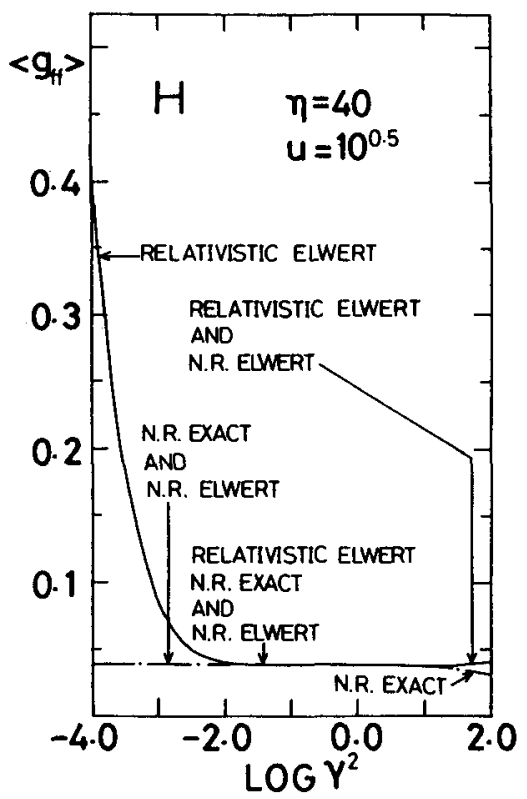

FIG. 2. Same as FIG. 1, but for $\eta=40$. 


\section{REFERENCES}

Itoh, N., Nakagawa,M., and Kohyama,Y. 1985, Ap.J., 294, 17. Bethe,H.A., and Heitler,W. 1934, Proc.Roy.Soc.London, A1 46,83 .

Elwert,G. 1939, Ann. Phys., 34, 178 .

Sommerfeld,A. 1953, A tombau und Spektrallinien ( Braunschweig:Vieweg), Vol. 2, Chap. 7 .

Karzas,W.J., and Latter,R. 1961, Ap.J.,Suppl ., 6, 167. Green,J.M. 1960, Fermi-Dirac Averages of the Free-Free Hydrogenic Gaunt Factor (RAND Corporation Rept. $\mathrm{RM}-2580-\mathrm{AEC}$ ) .

Berger,J.M. 1956, Ap.J., $124,550$.

Berger,J.M. 1957, Phys.Rev., 105, 35.

Elwert,G., and Haug,E. 1969, Phys.Rev., 183, 90.

Pratt,R.H., and Tseng,H.K. 1975, Phys.Rev., A11, 1797.

Nakagawa,M., Kohyama,Y., and Itoh,N. 1987, Ap.J., Suppl., 63,661 . 